\title{
A meta-analysis of risk factors for lymph node posterior to the right recurrent laryngeal nerve metastasis in papillary thyroid carcinoma
}

\author{
Tianhan Zhou ${ }^{1}$, Fan Wu ${ }^{1}$, Lingqian Zhao ${ }^{1}$, Kecheng Jiang ${ }^{1}$, Dingcun Luo ${ }^{2}$ \\ ${ }^{1}$ The Fourth School of Clinical Medicine, Zhejiang Chinese Medical University, Hangzhou, China; ${ }^{2}$ Department of Surgical Oncology, Affiliated \\ Hangzhou First People's Hospital, Zhejiang University School of Medicine, Hangzhou, China \\ Contributions: (I) Conception and design: T Zhou; (II) Administrative support: None; (III) Provision of study materials or patients: None; (IV) \\ Collection and assembly of data: T Zhou, F Wu; (V) Data analysis and interpretation: T Zhou, F Wu; (VI) Manuscript writing: All authors; (VII) \\ Final approval of manuscript: All authors. \\ Correspondence to: Dingcun Luo. Department of Surgical Oncology, Affiliated Hangzhou First People's Hospital, Zhejiang University School of \\ Medicine, Hangzhou, China. Email: 1dc65@163.com.
}

\begin{abstract}
Background: Whether the lymph node posterior to the right recurrent laryngeal nerve (LN-prRLN) should be dissected is still controversial. This meta-analysis aimed to assess the risk factors for LN-prRLN metastasis in papillary thyroid carcinoma (PTC).

Methods: We retrieved relevant studies published before May 2020 from the Wanfang Data, CNKI, PubMed, Embase, Web of Science and Cochrane Library databases. Heterogeneity was assessed with the Q-test and inconsistency index and sensitivity analysis and subgroup analysis were then used to find the source of heterogeneity. Begg test and generate a funnel chart to assess publication bias.
\end{abstract}

Results: We retrieved 236 articles, 14 articles were selected as the subjects of our research. Fourteen studies involving 10,580 patients were analysed in this study. The LN-prRLN metastasis rate was $9.22 \%$ (975/10,580). The results of the meta-analysis showed that sex $(\mathrm{P}<0.001)$, age $(\mathrm{P}<0.001)$, tumour size $(\mathrm{P}<0.001)$, multifocality $(\mathrm{P}<0.001)$, capsular invasion $(\mathrm{P}=0.04)$, extrathyroidal extension $(\mathrm{P}<0.001)$, superficial central lymph node $(\mathrm{VIa}-\mathrm{LN})$ metastasis $(\mathrm{P}<0.001)$, and lateral lymph node $(\mathrm{LLN})$ metastasis $(\mathrm{P}<0.001)$ were correlated with LN-prRLN metastasis.

Discussion: Male sex, age $\leq 45$ years, tumour size $>1 \mathrm{~cm}$, multifocality, capsular invasion or extrathyroidal extension, and VIa-LN metastasis or LLN metastasis in PTC patients were significant risk factors for predicting LN-prRLN metastasis. B ultrasound and CT scans are expected to predict LN-prRLN metastasis in the future.

Registration: This research is registered on the PROSPERO website (registration number: CRD42020200898).

Keywords: Papillary thyroid carcinoma (PTC); right recurrent laryngeal nerve; risk factor

Submitted Mar 18, 2021. Accepted for publication May 13, 2021.

doi: $10.21037 /$ gs-21-177

View this article at: https://dx.doi.org/10.21037/gs-21-177

^ ORCID: 0000-0003-0292-3346. 


\section{Introduction}

In recent years, thyroid carcinoma has exhibited the rapidest increase in incidence among solid tumours in China. Papillary thyroid carcinoma (PTC) is the most common pathological type of thyroid cancer (1). PTC has a good prognosis despite approximately $30 \%$ to $80 \%$ of patients having cervical lymph node metastasis at the time of diagnosis (2). Therefore, most guidelines for preoperative or intraoperative examination of the central lymph node positive recommend central lymph node dissection (CLND) (3). However, the lack of a uniform surgical approach may lead to postoperative PTC relapse, which requires additional surgery. Furthermore, reoperation may increase the difficulty of the surgical procedure and the risks of permanent hypoparathyroidism and recurrent laryngeal nerve (RLN) injury (4). Therefore, it is very important to emphasize standard and thorough CLND.

Although the American Thyroid Association (ATA) guidelines outline the anatomical boundaries of CLND in thyroid cancer, the guidelines only define the width of the central compartment and not the depth of the central compartment (5). Whether the lymph node posterior to the right recurrent laryngeal nerve (LN-prRLN) should be dissected is still controversial. On the one hand, some researchers suggest that there is no need for routine LN-prRLN dissection because of the low LN-prRLN metastasis rate, the complex surgical approach and the high complication rate. On the other hand, some scholars have pointed out that the LN-prRLN metastasis rate in PTC patients is as high as $2.74-38.27 \%(6,7)$. Incomplete resection of the LN-prRLN in the first surgery can make the residual lymph nodes difficult to excise in the second surgery and even render complete excision impossible. Therefore, we conducted a meta-analysis to investigate the risk factors related to LN-prRLN metastasis and provide reliable evidence-based support for LN-prRLN dissection.

We present the following article in accordance with the PRISMA reporting checklist (available at https://dx.doi. org/10.21037/gs-21-177).

\section{Methods}

\section{Literature searching}

The search databases included Wanfang Data, CNKI, PubMed, Embase, Web of Science, and Cochrane Library. The search time was limited to the establishment of the database until May 2020. Subject terms and keywords were used to search, and a search strategy was formulated according to the characteristics of the databases. Chinese search terms were as follows: "thyroid tumour" or "thyroid cancer" or "thyroid papillary tumour" or "thyroid papillary carcinoma" and "laryngeal nerve rear" or "tracheoesophageal" and "lymph node metastasis"; English search terms were as follows: "thyroid carcinoma" or "thyroid cancer" or "thyroid papillary carcinoma" or "thyroid papillary cancer" and "recurrent laryngeal nerve" or "inferior laryngeal nerve" or "paraoesophageal" and "lymphatic metastasis" or "lymph node metastasis".

\section{Study inclusion/exclusion criteria}

The inclusion criteria were as follows: (I) PTC was confirmed via a pathological examination; (II) the neoplastic lesions were located in the right or bilateral glands of the thyroid gland without isthmic lesions; (III) patients receiving at least right central lymph node dissection (including LN-prRLN dissection); and (IV) the included studies were prospective or retrospective. The exclusion criteria were as follows: (I) comments, case series, overviews and conference abstracts; (II) repeated publications.

\section{Data extraction}

Two authors (Tianhan Zhou and Fan $\mathrm{Wu}$ ) read the full text independently and extracted the relevant data. Clinical information, including the first author, research time, research centre (country), demographic data (sex, age), and pathological data (tumour size, multifocality, capsular invasion, extrathyroidal extension, and lymph node metastasis), was collected. In the entire study evaluation process, if the two authors had different viewpoints, a third author joined the discussion to obtain a common viewpoint.

\section{Statistical analysis}

To assess the quality of the included studies, the Methodological Index for Non-Randomized Studies (MINORS) (8) was applied to all of the included studies.

Review Manager software (RevMan version 5.3) was used to generate forest plots. This study included binary data, which were analysed by the OR value and $95 \%$ CI. The Q test and the $\mathrm{I}^{2}$ value were used to analyse the heterogeneity of the studies. When $\mathrm{P}<0.10$ or $\mathrm{I}^{2}>50 \%$, the random effects model was adopted; otherwise, the fixed effects model was used for data analysis. Stata software (version 14.1) was used 
Table 1 Information on the included studies

\begin{tabular}{|c|c|c|c|c|c|c|}
\hline Study & Country & Number (n) & LN-prRLN (+) & LN-prRLN (-) & Quality & Variable \\
\hline Chang, 2015 (6) & Korea & 5,556 & 148 & 5,408 & 17 & (1)(2)(3)(4)(6) \\
\hline Yuan, 2017 (7) & China & 81 & 31 & 50 & 17 & (1)(2)(3)(4)(5)(6) \\
\hline Wang, 2017 (9) & China & 305 & 34 & 271 & 17 & (1)(2)(4)(5) \\
\hline Zhang, 2018 (11) & China & 301 & 46 & 255 & 17 & (1)(3)(4)(6) \\
\hline Hou, 2019 (12) & China & 424 & 96 & 328 & 17 & (1) (2)(3)(4)(5)(7) (8) \\
\hline Zhang, 2016 (13) & China & 245 & 33 & 212 & 17 & (1)(2)(3)(4)(5)(7)(8) \\
\hline Zhu, 2019 (14) & China & 592 & 111 & 581 & 17 & (1)(3)(4)(6) \\
\hline Kim, 2012 (17) & Korea & 243 & 14 & 229 & 18 & (1)(3)(4)(6) \\
\hline Ito, 2013 (18) & Japan & 92 & 127 & 795 & 18 & (1)(4)(6) \\
\hline Yu, 2018 (19) & China & 829 & 158 & 671 & 17 & (1)(2)(3)(4)(5)(7)(8) \\
\hline
\end{tabular}

(1) sex, (2) age, (3) tumour size, (4) multifocality, (5) capsular invasion, (6) extrathyroidal extension, (7) LLN metastasis, (8) Vla-LN metastasis. LN-prRLN, lymph node posterior to the right recurrent laryngeal nerve.

to perform the Begg test and generate a funnel chart to assess publication bias. $\mathrm{P}>0.10$ indicated no publication bias.

\section{Results}

\section{Results of filtering literature}

A total of 236 articles were retrieved. After reading the titles and abstracts, we excluded articles that were not in accordance with the inclusion criteria, and 36 articles remained. Then, we screened the full texts, and 14 articles were ultimately included in this study $(4,6,7,9-19)$ (Table 1 ). The flowchart of article screening is shown in Figure 1. The above 14 articles were included in this study, and the Begg test and funnel charts were used to assess publication bias. The funnel chart shows that there was no publication bias in this meta-analysis $(\mathrm{P}=0.788$, Figure 2$)$.

\section{Characteristics of the included studies}

All of the included 14 articles, with publication dates ranging from 2009 to 2019, were based on nonrandomized studies. Basic information of 14 cohorts from these studies, giving a total of 10,580 cases, was included in the analysis.
The LN-prRLN metastasis rate was 9.22\% (975/10,580). Detailed information of each study is provided in Table 1 . The MINORS criteria (Table 2) were applied to all 14 studies.

\section{Risk factors for $L N-p r R L N$}

\section{Sex}

Fourteen studies $(4,6,7,9-19)$ involving 2,249 males and 8,331 females were included. In the comparison of sex, our results showed that male sex was a risk factor affecting $\mathrm{LN}$ prRLN metastasis (OR $=1.47,95 \%$ CI: $1.26-1.72, \mathrm{P}<0.001$, $\mathrm{I}^{2}=63$, Figure 3). There was large heterogeneity in this group of studies. When the study by Yu et al. was excluded, $\mathrm{I}^{2}=0 \%$, indicating that the study by $\mathrm{Yu}$ et al. was the main source of heterogeneity.

\section{Age}

Seven articles $(6,7,9-12,19)$ were included in this study; in these articles, 3,768 patients were aged $\leq 45$ years, and 3,956 patients were aged $\geq 45$ years. Our results showed significant differences when the datasets were combined for the comparison of age (OR $=1.63,95 \%$ CI: $1.23-2.14, \mathrm{P}=0.003$, $\mathrm{I}^{2}=44 \%$, Figure 4$)$. When the study by Hou et al. was 


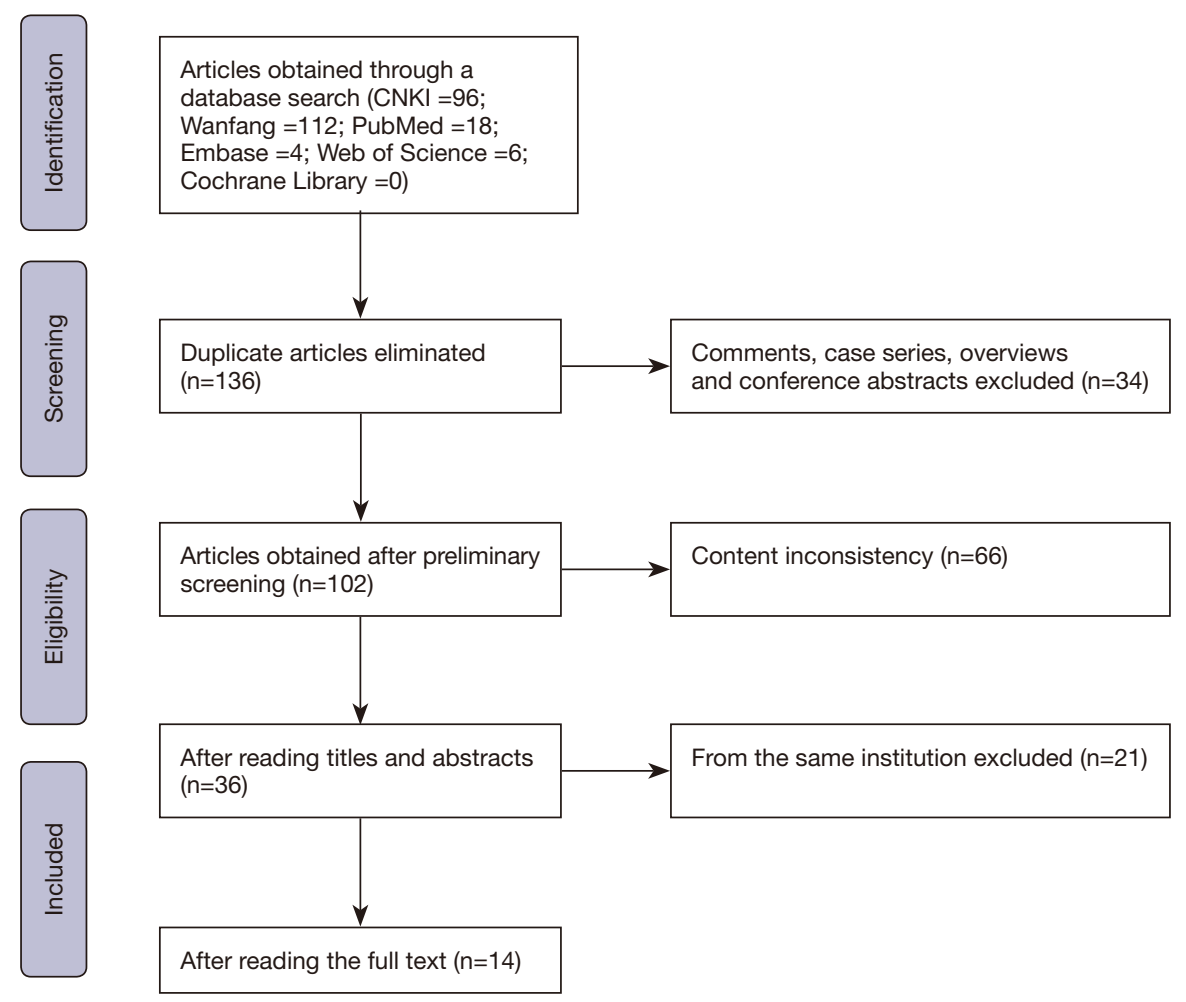

Figure 1 The flowchart of article screening.

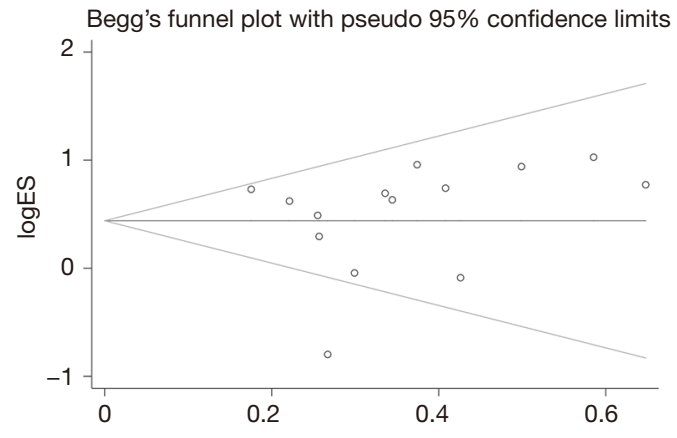

Figure 2 The funnel chart of risk of bias.

excluded, the heterogeneity decreased to $\mathrm{I}^{2}=0 \%$; thus, the study by Hou $e t a l$. was the main source of heterogeneity.

\section{Tumour size}

Twelve articles $(4,6,7,10-17,19)$ were included in this study. In total, 3,368 patients had a tumour size $>1 \mathrm{~cm}$, and 6,012 patients had a tumour size $\leq 1 \mathrm{~cm}$. The analysis showed that tumour size is a risk factor for LN-prRLN metastasis. The probability of LN-prRLN in patients with a tumour diameter $>1 \mathrm{~cm}$ was 3.40 times higher that of patients with a tumour diameter $\leq 1 \mathrm{~cm}(\mathrm{OR}=3.40,95 \% \mathrm{CI}$ : $2.90-3.98, \mathrm{P}<0.001, \mathrm{I}^{2}=24 \%$, Figure 5).

\section{Multifocality}

Twelve studies (4,6,7,9-14,17-19) including 3,463 patients with multifocal disease and 6,755 patients with unifocal disease were analysed in this study. Our results showed significant differences between the two groups (OR $=2.08,95 \%$ CI: $1.61-2.68, \mathrm{P}<0.001, \mathrm{I}^{2}=58 \%$, Figure 6). Heterogeneity $\mathrm{t}$ was tested in two different groups (Chinese studies and other Asian studies) through subgroup analysis. The subgroup analysis showed that heterogeneity was lower and that the overall effect remained significant (OR $=2.07,95 \%$ CI: $1.56-2.75, \mathrm{P}<0.001, \mathrm{I}^{2}=0 \%$, Figure 7). Thus, regional differences were the main source of heterogeneity in this group.

\section{Capsular invasion}

Seven articles $(4,7,9,12,13,15,19)$ including 623 patients with capsular invasion and 1,691 patients without capsular invasion were analysed in this study. In the analysis of 
Table 2 The MINORS score of each study

\begin{tabular}{|c|c|c|c|c|c|c|c|c|c|c|c|c|c|}
\hline Study & \multicolumn{12}{|c|}{ Methodological index for non-randomized studies } & Total \\
\hline Luo, 2017 & 2 & 2 & 2 & 2 & 0 & 2 & 2 & 0 & 2 & 2 & 0 & 2 & 18 \\
\hline Chang, 2015 & 2 & 2 & 1 & 2 & 0 & 2 & 2 & 0 & 2 & 2 & 0 & 2 & 17 \\
\hline Yuan, 2017 & 2 & 2 & 1 & 2 & 0 & 2 & 2 & 0 & 2 & 2 & 0 & 2 & 17 \\
\hline Zhang, 2014 & 2 & 2 & 2 & 2 & 0 & 2 & 2 & 0 & 2 & 2 & 0 & 2 & 18 \\
\hline Zhang, 2018 & 2 & 2 & 1 & 2 & 0 & 2 & 2 & 0 & 2 & 2 & 0 & 2 & 17 \\
\hline Hou, 2019 & 2 & 2 & 1 & 2 & 0 & 2 & 2 & 0 & 2 & 2 & 0 & 2 & 17 \\
\hline Zhang, 2016 & 2 & 2 & 1 & 2 & 0 & 2 & 2 & 0 & 2 & 2 & 0 & 2 & 17 \\
\hline Bae, 2012 & 2 & 2 & 2 & 2 & 0 & 2 & 2 & 0 & 2 & 2 & 0 & 2 & 18 \\
\hline Kim, 2012 & 2 & 2 & 2 & 2 & 0 & 2 & 2 & 0 & 2 & 2 & 0 & 2 & 18 \\
\hline Ito, 2013 & 2 & 2 & 2 & 2 & 0 & 2 & 2 & 0 & 2 & 2 & 0 & 2 & 18 \\
\hline Yu, 2018 & 2 & 2 & 1 & 2 & 0 & 2 & 2 & 0 & 2 & 2 & 0 & 2 & 17 \\
\hline
\end{tabular}

1, A stated aim of the study; 2, inclusion of consecutive patients; 3, prospective collection of data; 4, endpoint appropriate to the study aim; 5 , unbiased evaluation of endpoints; 6 , follow-up period appropriate to the major endpoint; 7 , loss to follow up not exceeding $5 \%$; 8 , a control group having the gold standard intervention; 9, Contemporary groups; 10, baseline equivalence of groups; 11, prospective calculation of the sample size; 12 , statistical analyses adapted to the study design. MINORS, methodological index for non-randomized studies.

\begin{tabular}{|c|c|c|c|c|c|c|c|c|c|c|}
\hline \multirow[b]{2}{*}{ Study or subgroup } & \multicolumn{2}{|c|}{ Male } & \multicolumn{3}{|c|}{ Female } & \multirow{2}{*}{$\begin{array}{c}\text { Odds Ratio } \\
\text { M-H, Fixed, 95\% Cl }\end{array}$} & \multirow{2}{*}{\multicolumn{3}{|c|}{$\begin{array}{c}\text { Odds Ratio } \\
\text { M-H, Fixed, } 95 \% \mathrm{Cl}\end{array}$}} & \\
\hline & Events & Total & Events & Total & I Weight & & & & & \\
\hline Bae.S.Y 2012 & 15 & 83 & 30 & 286 & $4.5 \%$ & $1.88[0.96,3.70]$ & & & & \\
\hline Chang.H 2015 & 52 & 1171 & 96 & 4385 & $15.7 \%$ & $2.08[1.47,2.93]$ & & & & \\
\hline Hou.J.Z 2019 & 29 & 109 & 67 & 315 & $10.3 \%$ & $1.34[0.81,2.22]$ & & & & \\
\hline Ito.Y 2013 & 23 & 118 & 104 & 804 & $8.7 \%$ & $1.63[0.99,2.69]$ & & & & \\
\hline Kim.Y.S 2012 & 5 & 43 & 9 & 200 & $1.1 \%$ & $2.79[0.89,8.80]$ & & & & \\
\hline Lee.B.J 2009 & 4 & 21 & 10 & 102 & $1.1 \%$ & $2.16[0.61,7.71]$ & & & & \\
\hline Luo.D.C 2017 & 17 & 68 & 34 & 238 & $4.6 \%$ & $2.00[1.04,3.86]$ & & & & \\
\hline Wang.F.Q 2017 & 15 & 78 & 19 & 227 & $3.2 \%$ & $2.61[1.25,5.43]$ & & & & \\
\hline Yu.Q.A 2018 & 18 & 167 & 140 & 662 & $20.4 \%$ & $0.45[0.27,0.76]$ & & & & \\
\hline Yuan.J.L2017 & 13 & 24 & 18 & 57 & $2.0 \%$ & $2.56[0.96,6.81]$ & & & & \\
\hline Zhang.L 2016 & 11 & 52 & 22 & 194 & $3.0 \%$ & $2.10[0.94,4.67]$ & & & & \\
\hline Zhang.P.Y2014 & 21 & 79 & 56 & 204 & $9.3 \%$ & $0.96[0.53,1.72]$ & & & & \\
\hline Zhang.T 2018 & 8 & 71 & 28 & 230 & $4.8 \%$ & $0.92[0.40,2.11]$ & & & & \\
\hline Zhu.J 2019 & 43 & 165 & 68 & 427 & $11.4 \%$ & $1.86[1.21,2.87]$ & & & & \\
\hline Total $(95 \% \mathrm{Cl})$ & & 2249 & & 8331 & $100.0 \%$ & $1.47[1.26,1.72]$ & & & & \\
\hline Total events & 274 & & 701 & & & & & & & \\
\hline \multicolumn{7}{|c|}{ Heterogeneity: $\mathrm{Chi}^{2}=35.46, \mathrm{df}=13(P=0.0007) ; \mathrm{I}^{2}=63 \%$} & 0.2 & 0.5 & 2 & 5 \\
\hline \multicolumn{7}{|c|}{ Test for overall effect: $Z=4.86(P<0.00001)$} & 0.2 & Female & Male & \\
\hline
\end{tabular}

Figure 3 Forest plot for LN-prRLN metastasis according to sex. LN-prRLN, lymph node posterior to the right recurrent laryngeal nerve. 


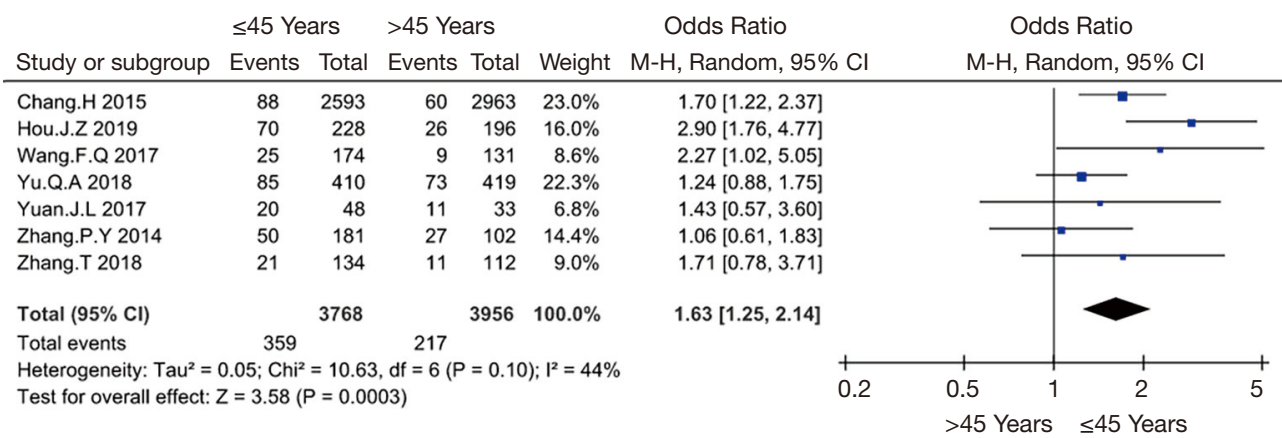

Figure 4 Forest plot for LN-prRLN metastasis according to age. LN-prRLN, lymph node posterior to the right recurrent laryngeal nerve.

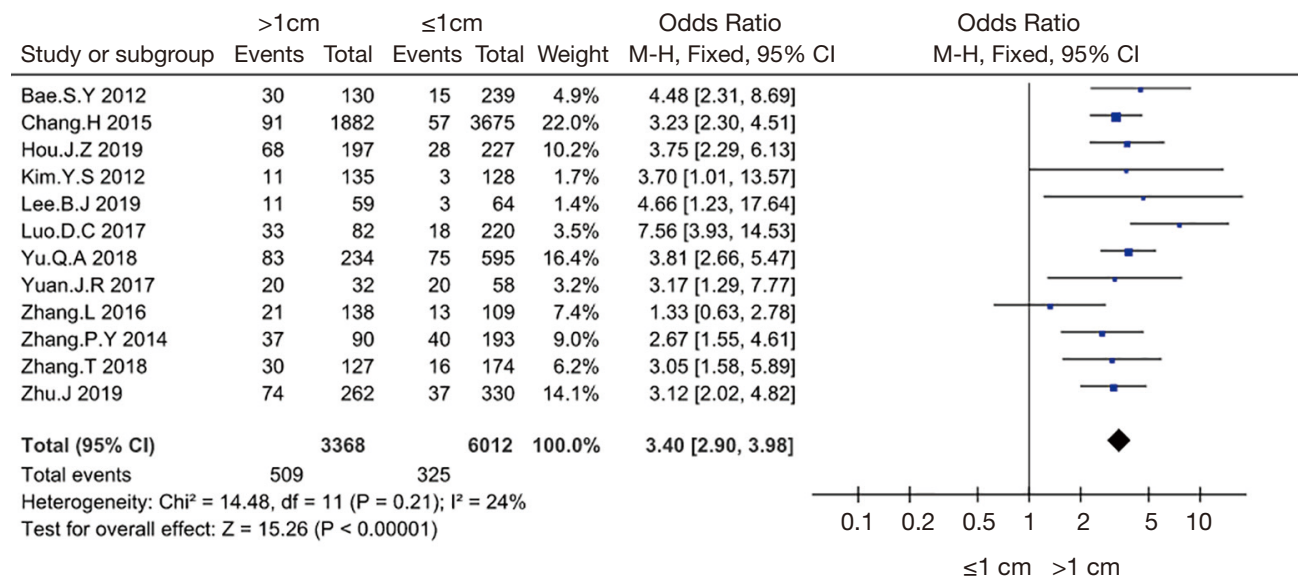

Figure 5 Forest plot for LN-prRLN metastasis according to tumour size. LN-prRLN, lymph node posterior to the right recurrent laryngeal nerve.

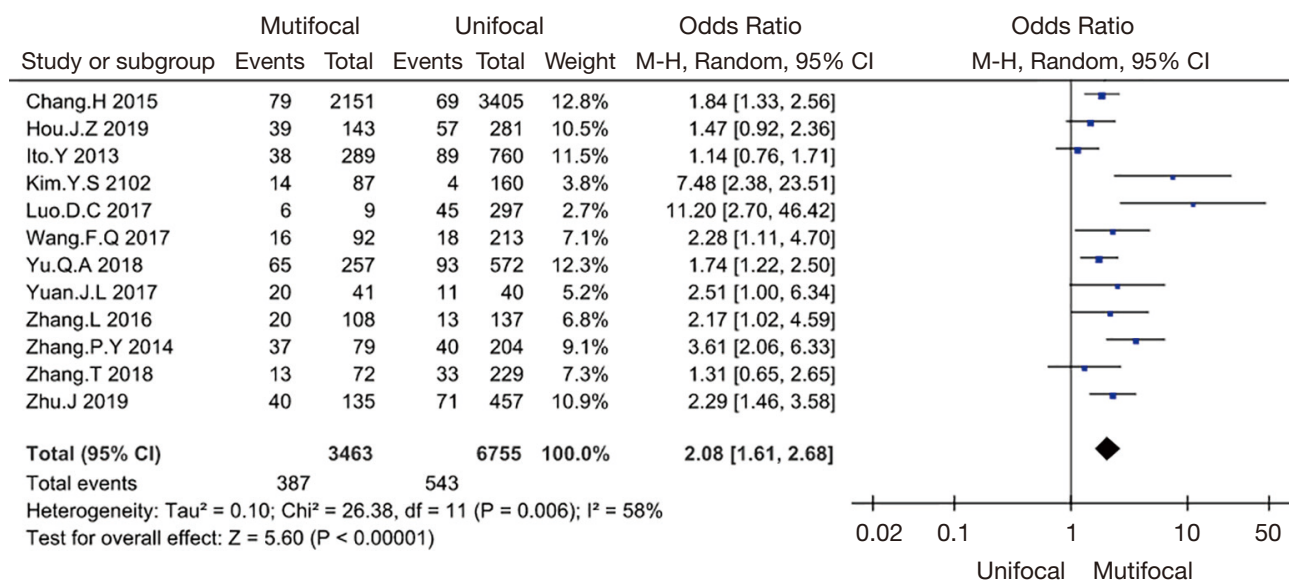

Figure 6 Forest plot for LN-prRLN metastasis according to multifocality. LN-prRLN, lymph node posterior to the right recurrent laryngeal nerve. 


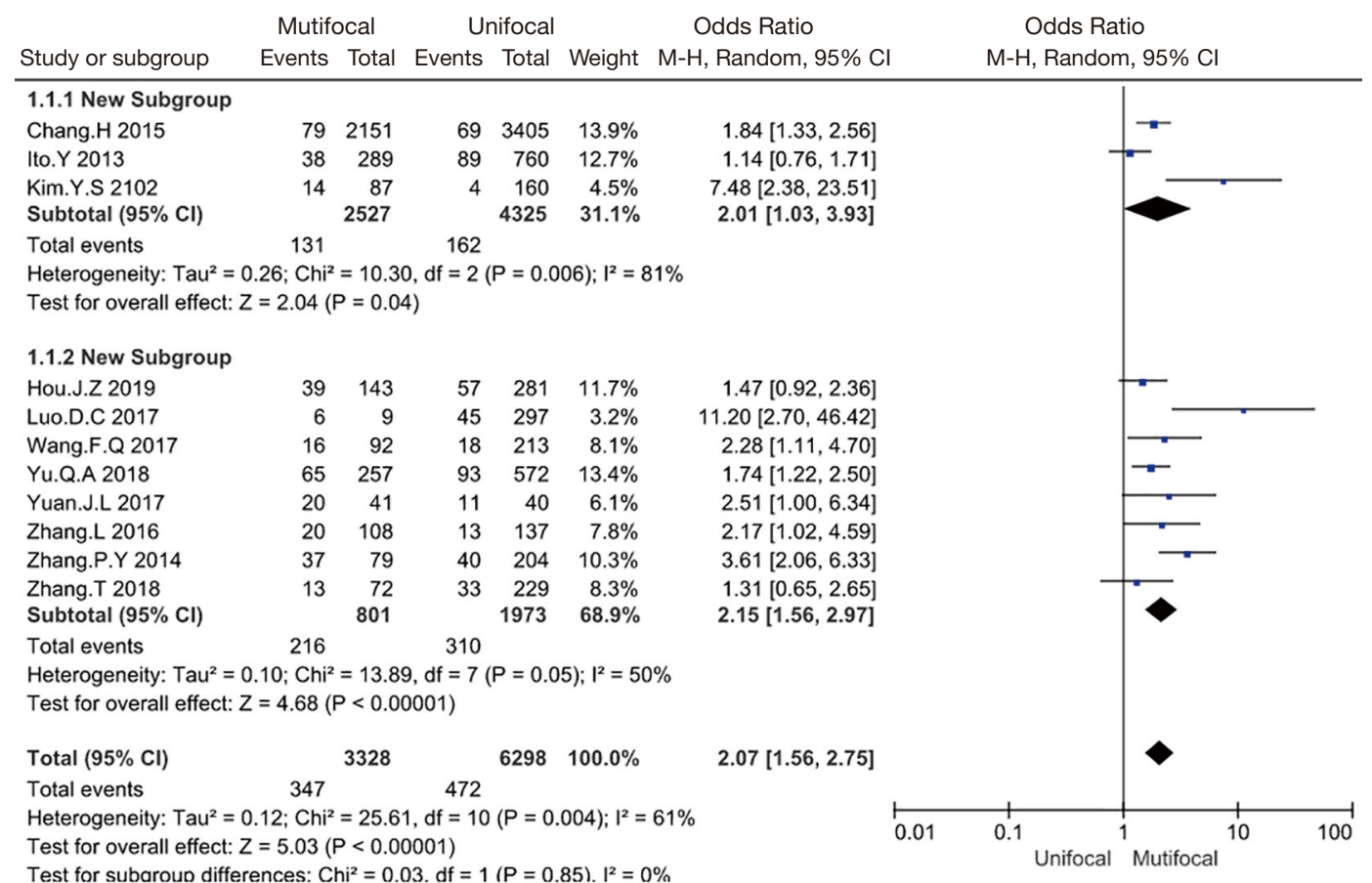

Figure 7 Forest plot for LN-prRLN metastasis according to multifocality by subgroup analysis. LN-prRLN, lymph node posterior to the right recurrent laryngeal nerve.

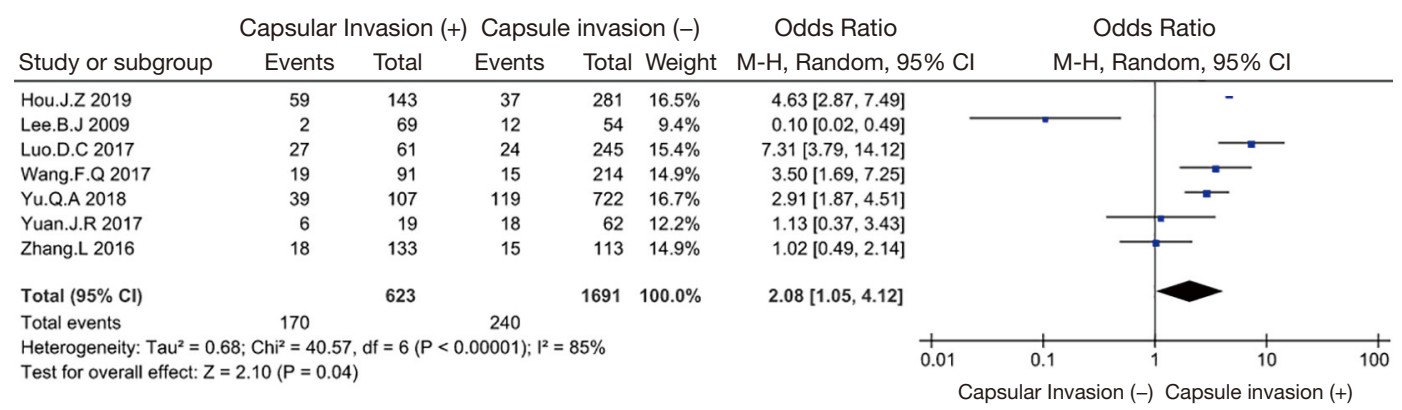

Figure 8 Forest plot for LN-prRLN metastasis according to capsular invasion. LN-prRLN, lymph node posterior to the right recurrent laryngeal nerve.

risk factors for capsular invasion, there was a significant difference regarding capsular invasion between the two groups. When PTC patients had capsular invasion, LNprRLN metastasis was 2.08 times higher that of PTC patients without capsular invasion $(\mathrm{OR}=2.08,95 \% \mathrm{CI}$ : 1.05-4.12, $\mathrm{P}=0.04, \mathrm{I}^{2}=85 \%$, Figure 8).

\section{Extrathyroidal extension}

Seven articles $(4,6,7,10,11,14,17,18)$ involving 3,966 patients with extrathyroidal extension and 3,891 patients without extrathyroidal extension were included in this study. In the analysis of risk factors for extrathyroidal extension, extrathyroidal extension was closely related to LN-prRLN metastasis in PTC (OR =3.48, 95\% CI: 2.02-6.00, $\mathrm{P}<0.001$, $\mathrm{I}^{2}=72 \%$, Figure 9). When the study by Zhang et al. was excluded, the heterogeneity decreased to $\mathrm{I}^{2}=0 \%$; thus, the study by Zhang et al. was the main source of heterogeneity.

\section{Central superficial lymph node (VIa-LN) metastasis}

Six articles $(4,10,12-14,19)$ on 1,175 patients with VIa-LN metastasis and 1,505 patients without VIa-LN metastasis were included. In the analysis of risk factors for VIa-LN 


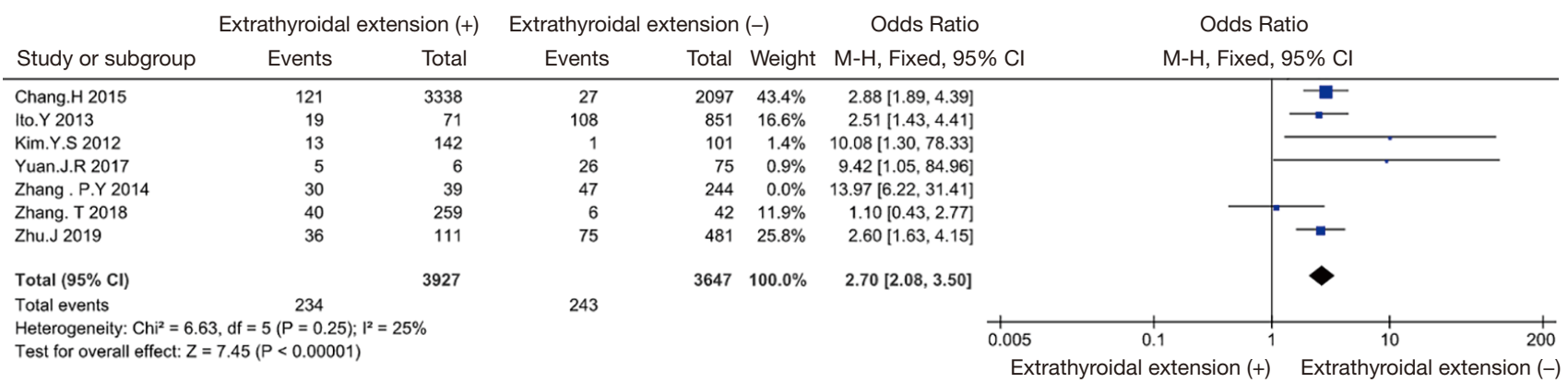

Figure 9 Forest plot for LN-prRLN metastasis according to extrathyroidal extension. LN-prRLN, lymph node posterior to the right recurrent laryngeal nerve.

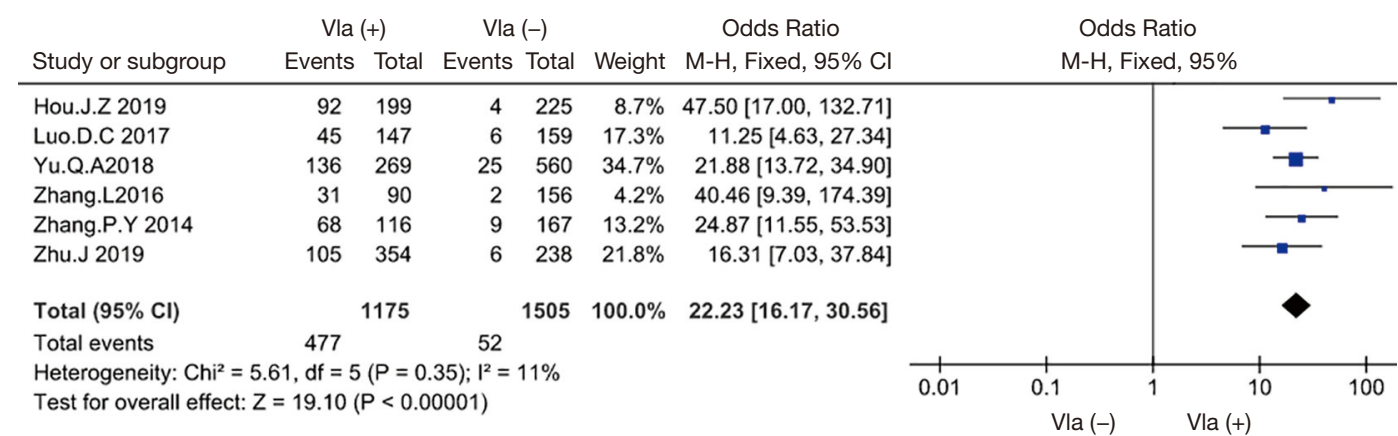

Figure 10 Forest plot for LN-prRLN metastasis according to VIa-LN metastasis. LN-prRLN, lymph node posterior to the right recurrent laryngeal nerve.

metastasis, the results indicated that VIa-LN metastasis was significantly associated with LN-prRLN. When patients had lymph node metastasis in the central area, the probability of LN-prRLN metastasis was 22.23 times higher, and this group had a low heterogeneity $(\mathrm{OR}=22.23$, 95\% CI: $16.17-30.56, \mathrm{P}<0.001, \mathrm{I}^{2}=11 \%$, Figure 10$)$.

\section{Lateral lymph node (LLN) metastasis}

Eight studies $(4,10,12-16,19)$ involving 616 patients with LLN metastasis and 2,554 patients without LLN metastasis were analysed. In the analysis of risk factors for LLN metastasis, the results showed a significant difference between the two groups $(\mathrm{OR}=8.27,95 \% \mathrm{CI}: 6.37-10.75$, $\mathrm{P}<0.001, \mathrm{I}^{2}=22 \%$, Figure 11).

\section{Discussion}

In 2009, Lee et al. in Korea initially reported the relationship between PTC and LN-prRLN metastasis (15). Subsequent research by Ito et al. in Japan confirmed that the clinicopathological characteristics of PTC are related to LN-prRLN metastasis (18). We found that $9.22 \%$ (975/10,580) of PTC patients had LN-prRLN metastasis, which falls into the range of the metastatic rate reported previously (2.74-38.27\%). Therefore, PTC still has a high probability of LN-prRLN metastasis, and some relevant clinicopathological features of thyroid cancer can increase the risk of LN-prRLN metastasis. However, a systematic review was lacking, and there was no evidence of risk factors to predict LN-prRLN metastasis.

The relationship between demographic factors and LN-prRLN metastasis remains controversial. Among the 14 studies included in this study, those by Wang et al. and Kim et al. suggested that male patients were more likely to develop LN-prRLN metastasis than female patients $(9,17)$. After the data were combined, the results of the meta-analysis showed that sex and LN-prRLN metastasis were correlated; this correlation was likely related to the increased sample size. The 8th edition of the AJCC guidelines raised the age stratification to 55 years, but most 


\begin{tabular}{|c|c|c|c|c|c|c|c|c|c|c|}
\hline \multirow[b]{2}{*}{ Study or subgroup } & \multicolumn{2}{|c|}{ LLN(+) } & \multicolumn{2}{|c|}{ LNN(-) } & \multirow[b]{2}{*}{ Weight } & \multirow{2}{*}{$\begin{array}{l}\text { Odds Ratio } \\
\mathrm{M}-\mathrm{H}, \text { Random, } 95 \% \mathrm{Cl}\end{array}$} & \multirow{2}{*}{\multicolumn{3}{|c|}{$\begin{array}{l}\text { Odds Ratio } \\
\mathrm{M}-\mathrm{H}, \text { Random, } 95 \% \mathrm{Cl}\end{array}$}} & \\
\hline & Events & Total & Events & Total & & & & & & \\
\hline Bae.S.Y 2012 & 20 & 61 & 25 & 308 & $12.0 \%$ & $5.52[2.82,10.82]$ & & & & \\
\hline Hou.J.Z 2019 & 49 & 77 & 47 & 347 & $16.0 \%$ & $11.17[6.40,19.49]$ & & & & \\
\hline Lee.B.J 2009 & 7 & 17 & 7 & 104 & $4.2 \%$ & $9.70[2.83,33.30]$ & & & & \\
\hline Luo.D.C 2017 & 28 & 47 & 23 & 259 & $10.7 \%$ & $15.12[7.34,31.16]$ & & & & \\
\hline Yu.Q.A 2018 & 54 & 89 & 104 & 740 & $20.0 \%$ & $9.44[5.88,15.14]$ & & & & - \\
\hline Zhang.L 2016 & 18 & 54 & 15 & 192 & $9.5 \%$ & $5.90[2.72,12.78]$ & & & & \\
\hline Zhang.P.Y 2014 & 16 & 28 & 61 & 255 & $9.0 \%$ & $4.24[1.90,9.45]$ & & & & \\
\hline Zhu.J 2019 & 88 & 243 & 23 & 349 & $18.7 \%$ & $8.05[4.89,13.23]$ & & & & \\
\hline Total $(95 \% \mathrm{Cl})$ & & 616 & & 2554 & $100.0 \%$ & $8.27[6.37,10.74]$ & & & & \\
\hline Total events & 280 & & 305 & & & & & & & \\
\hline \multicolumn{7}{|c|}{$\begin{array}{l}\text { Heterogeneity: } \mathrm{Tau}^{2}=0.03 ; \mathrm{Chi}^{2}=8.95, \mathrm{df}=7(\mathrm{P}=0.26) ; \mathrm{I}^{2}=22 \% \\
\text { Test for overall effect: } Z=15.85(P<0.00001)\end{array}$} & 0.05 & 0.2 & $1 \quad 5$ & 20 \\
\hline
\end{tabular}

Figure 11 Forest plot for LN-prRLN metastasis according to LNM. LN-prRLN, lymph node posterior to the right recurrent laryngeal nerve.

of the studies included used 45 years as the classification standard. Patients aged $\leq 45$ years were more likely to develop LN-prRLN metastasis than those aged $>45$ years, which is consistent with the results of the studies by two scholars, including Zhang et al. $(11,12)$. In our study, the OR value of demographic factor is smallest, but its $95 \%$ confidence interval is short, which indicates the result has a certain accuracy. Therefore, sex and age are risk factors that affect LN-prRLN metastasis.

Tumour size and multifocality were also correlated with LN-prRLN metastasis. In this study, tumour size $>1 \mathrm{~cm}$ was used as the classification criterion. The univariate analysis of 12 studies suggested that tumour size is related to LN-prRLN metastasis, while the multivariate analysis of 5 studies showed that tumour size is still an independent risk factor for LN-prRLN metastasis. Multifocality is not only related to LN-prRLN metastasis but also closely correlated with the prognosis of PTC patients. A previous meta-analysis indicated that multifocality is an important risk factor for disease progression and increases the risk of disease recurrence (20). In our study, we further performed subgroup analysis and our result showed that region was the source of multifocal heterogeneity. The possible reason is that Asian countries such as South Korea have higher levels of iodine intake than China (21). If the tumour size is $>1 \mathrm{~cm}$ or multifocal disease is present, it is necessary to perform LN-prRLN dissection.

In fact, thyroid capsular invasion and extrathyroidal extension are two forms that describe the extent to which the tumour invades surrounding tissues. When PTC invades the capsule or extrathyroidal organs, it often increases the risk of LN-prRLN metastasis. According to previous reports, the ten-year survival rate of patients decreases from $99.3 \%$ to $63 \%$, if the tumour invades or breaks through the capsule (22). Succinctly, LN-prRLN dissection should be performed to reduce the risk of incomplete surgical resection if there is a visible representation of capsular invasion or extrathyroidal extension (23).

Our previous results suggested that the presence of VIaLN metastasis, >2 metastatic LNs and metastatic LNs > $0.45 \mathrm{~cm}$ in size demonstrated a significant association with LN-prRLN metastasis (24). Although the LLN is the second station for $\mathrm{LN}$ metastasis, the $\mathrm{OR}$ value of lateral LN metastasis was significant. The risk factor of LN metastasis had the highest OR value among all risk factors. In our study, the OR value of VIa-LN metastasis was higher than that of LLN metastasis. The reason is thought to be the anatomy of the central region and lymph node drainage. In this study, it was proven again that VIa-LN metastasis and LLN metastasis are independent risk factors for LNprRLN metastasis.

Apart from the above mentioned indicators, some studies have described the use of $\mathrm{B}$ ultrasound and computed tomography (CT) scans to evaluate the status of $\mathrm{LN}$ prRLN metastasis. Zhang et al. reported that a CT value exceeding $72 \mathrm{hu}$ often indicates the presence of lymph node metastasis (11). In addition, the effect of B ultrasound in assessing LN-prRLN metastasis is conservative. According to a study by Qu et al., the sensitivity and specificity of ultrasound are $11.1 \%$ and $95.8 \%$ (11). Only two studies proposed imaging indicators to assess LN-prRLN metastasis, so they were not included in this study. In addition, more data are needed to prove this hypothesis.

This is a meta-analysis with the largest sample size so far, 
but there are still some limitations. (I) Risk factors including tumor size, extrathyroidal extension, and central LN metastasis have been shown to correlate with LN-prRLN metastasis in previous studies. And we expect to find more correlations between LN-prRLN metastasis and risk factors such as imaging omics. (II) More prospective studies are expected to be included to improve the credibility of the meta-analysis.

\section{Conclusions}

In conclusion, male sex, age $\leq 45$ years, tumour size $>1 \mathrm{~cm}$, multifocality, capsular invasion or extrathyroidal extension, and VIa-LN metastasis or LLN metastasis in PTC patients are significant risk factors for predicting LN-prRLN metastasis. B ultrasound and CT scans are expected to predict LN-prRLN metastasis in the future.

\section{Acknowledgments}

The authors thank SNAS for the help in language editing. Funding: The Project of Medical Scientific and Technology Program in Hangzhou (grant number A20200432); Science Research Program of Hangzhou (20180533B39).

\section{Footnote}

Reporting Checklist: The authors have completed the PRISMA reporting checklist. Available at https://dx.doi. org/10.21037/gs-21-177

Peer Review File: Available at https://dx.doi.org/10.21037/ gs-21-177

Conflicts of Interest: All authors have completed the ICMJE uniform disclosure form (available at https://dx.doi. org/10.21037/gs-21-177). The authors have no conflicts of interest to declare.

Ethical Statement: The authors are accountable for all aspects of the work in ensuring that questions related to the accuracy or integrity of any part of the work are appropriately investigated and resolved.

Open Access Statement: This is an Open Access article distributed in accordance with the Creative Commons Attribution-NonCommercial-NoDerivs 4.0 International License (CC BY-NC-ND 4.0), which permits the noncommercial replication and distribution of the article with the strict proviso that no changes or edits are made and the original work is properly cited (including links to both the formal publication through the relevant DOI and the license). See: https://creativecommons.org/licenses/by-nc-nd/4.0/.

\section{References}

1. Booth CM, Karim S, Mackillop WJ. Real-world data: towards achieving the achievable in cancer care. Nat Rev Clin Oncol 2019;16:312-25.

2. Xue XC, Ding JW, Peng Y et al. Clinical research of the lymph node dissection posterior to the right recurrent laryngeal nerve in papillary thyroid carcinoma. Chinese Archives of Otolaryngology-Head and Neck Surgery 2018;25:61-5.

3. Haugen BR, Alexander EK, Bible KC, et al. 2015 American Thyroid Association Management Guidelines for Adult Patients with Thyroid Nodules and Differentiated Thyroid Cancer: The American Thyroid Association Guidelines Task Force on Thyroid Nodules and Differentiated Thyroid Cancer. Thyroid 2016;26:1-133.

4. Luo DC, Xu XC, Ding JW et al. Clinical value and indication for the dissection of lymph nodes posterior to the right recurrent laryngeal nerve in papillary thyroid carcinoma. Oncotarget 2017;8:79897-905.

5. American Thyroid Association Surgery Working Group; American Association of Endocrine Surgeons; American Academy of Otolaryngology-Head and Neck Surgery; American Head and Neck Society, Carty SE, Cooper DS, Doherty GM, et al. Consensus statement on the terminology and classification of central neck dissection for thyroid cancer. Thyroid 2009;19:1153-8.

6. Chang H, Yoo RN, Kim SM et al. The Clinical Significance of the Right Para-Oesophageal Lymph Nodes in Papillary Thyroid Cancer. Yonsei Med J 2015;56:1632-7.

7. Yuan J, Li J, Chen X, et al. Predictors of lymph nodes posterior to the right recurrent laryngeal nerve metastasis in patients with papillary thyroid carcinoma: A retrospective study. Medicine (Baltimore) 2017;96:e7908.

8. Slim K, Nini E, Forestier D, et al. Methodological index for non-randomized studies (MINORS): development and validation of a new instrument. ANZ J Surg 2003:73:712-6.

9. Wang FQ, An YS, Ren ZF, et al. Study of the right paraesophageal node dissection for $\mathrm{cN} 0$ stage papillary thyroid microcarcinoma. Clinical Medicine of China 2017;33:981-4. 
10. Zhang $\mathrm{P}$, Zhang B, Pu JL, et al. Prospective analysis of the risk factors and clinical indications of dissection of lymph node posterior to right recurrent laryngeal nerve in 283 cases of papillary thyroid carcinoma. Chinese Journal of Oncology 2014;36:109-14.

11. Zhang T, Qu Y, He L, et al. Risk factors and preoperative evaluation of lymph nodes posterior to right recurrent laryngeal nerve metastasis in thyroid papillary carcinoma. National Medical Journal of China 2018;98:1775-9.

12. Hou JZ, Zhang YC, et al. Risk factors for lymph node posterior right recurrent laryngeal nerve metastasis of papillary thyroid carcinoma. Journal of Surgery Concepts \& Practice 2019;24:507-11.

13. Zhang L, Liu H, Xie Y, et al. Risk factors and indication for dissection of right paraesophageal lymph node metastasis in papillary thyroid carcinoma. Eur J Surg Oncol 2016;42:81-6.

14. Zhu J, Huang R, Hu D, et al. Individualized Prediction of Metastatic Involvement of Lymph Nodes Posterior To The Right Recurrent Laryngeal Nerve In Papillary Thyroid Carcinoma. Onco Targets Ther 2019;12:9077-84.

15. Lee BJ, Lee JC, Wang SG, et al. Metastasis of right upper para-esophageal lymph nodes in central compartment lymph node dissection of papillary thyroid cancer. World J Surg 2009;33:2094-8.

16. Bae SY, Yang JH, Choi MY, et al. Right paraesophageal lymph node dissection in papillary thyroid carcinoma. Ann Surg Oncol 2012;19:996-1000.

17. Kim YS, Park WC. Clinical predictors of right upper

Cite this article as: Zhou T, Wu F, Zhao L, Jiang K, Luo D. A meta-analysis of risk factors for lymph node posterior to the right recurrent laryngeal nerve metastasis in papillary thyroid carcinoma. Gland Surg 2021;10(6):1841-1851. doi: 10.21037/gs-21177 paraesophageal lymph node metastasis from papillary thyroid carcinoma. World J Surg Oncol 2012;10:164.

18. Ito Y, Fukushima M, Higashiyama T, et al. Incidence and predictors of right paraesophageal lymph node metastasis of N0 papillary thyroid carcinoma located in the right lobe. Endocr J 2013;60:389-92.

19. Yu QA, Ma DK, Liu KP, et al. Clinicopathologic risk factors for right paraesophageal lymph node metastasis in patients with papillary thyroid carcinoma. J Endocrinol Invest 2018;41:1333-8.

20. Joseph KR, Edirimanne S, Eslick GD. Multifocality as a prognostic factor in thyroid cancer: A meta-analysis. Int J Surg 2018;50:121-5.

21. Machens A, Dralle H. Simultaneous medullary and papillary thyroid cancer: a novel entity? Ann Surg Oncol 2012;19:37-44.

22. Kamaya A, Tahvildari AM, Patel BN, et al. Sonographic Detection of Extracapsular Extension in Papillary Thyroid Cancer. J Ultrasound Med 2015;34:2225-30.

23. Luo Y, Xu XC, Shen J, et al. Model of lymph node metastasis posterior to the right recurrent laryngeal nerve in papillary thyroid carcinoma. Cancer Manag Res 2018;10:2449-55.

24. Shi JJ, Xu XC, Wu J, et al. Clinical research about level VI-1 lymph nodes and the lymph nodes posterior to the right recurrent laryngeal nerve of the papillary thyroid carcinoma J. Chinese Archives of Otolaryngology-Head and Neck Surgery 2017;24:233-6. 